\title{
ON THE CHROMATIC NUMBER OF PLANE TILINGS
}

\author{
D. COULSON
}

(Received 17 June 2002; revised 25 June 2003)

\author{
Communicated by C. F. Miller
}

\begin{abstract}
It is known that $4 \leq \chi\left(\mathbb{R}^{2}\right) \leq 7$, where $\chi\left(\mathbb{R}^{2}\right)$ is the number of colours necessary to colour each point of Euclidean 2-space so that no two points lying distance 1 apart have the same colour. Any lattice-sublattice colouring scheme for $\mathbb{R}^{2}$ must use at least 7 colours to have an excluded distance. This article shows that at least 6 colours are necessary for an excluded distance when convex polygonal tiles (all with area greater than some positive constant) are used as the colouring base.
\end{abstract}

2000 Mathematics subject classification: primary 05B45, 52C20; secondary 05B40, 52C15.

\section{Introduction to the colouring problem}

The chromatic number $\chi\left(\mathbb{R}^{n}\right)$ of $\mathbb{R}^{n}$ is defined to be the minimum number of colours (sets $S_{i}$ ) necessary to colour $\mathbb{R}^{n}$ (such that $\bigcup_{i=1}^{x} S_{i}=\mathbb{R}^{n}$ ) so that the unit distance is excluded between points of the same colour $\left(\|x-y\| \neq 1\right.$ for all $x, y \in S_{i}$ for all $i$ ). Clearly any colouring with an excluded distance can be rescaled so that 1 is the excluded distance.

It is known that in the euclidean plane $\mathbb{R}^{2}$

$$
4 \leq \chi\left(\mathbb{R}^{2}\right) \leq 7 .
$$

The lower bound established by Raiskii [2] or the Moser spindle (see Figure 1) which needs at least 4 colours to colour it with an excluded distance.

The upper bound of 7 is established by a colouring based on congruent planefilling regular hexagonal cells (see Figure 2). This is an example of a lattice-sublattice colouring scheme where points in common Voronoi regions (also known as Delaunney

(C) 2004 Australian Mathematical Society $1446-7887 / 04 \$ A 2.00+0.00$ 
regions) are coloured the same according to what coset of the lattice/sublattice the central vertex is in (see Figure 2). It is known that we need at least 7 colours for a distance excluded colouring using lattice-sublattice colouring schemes (more generally in $n$-space $2^{n+1}-1$ colours are needed) see Coulson [1].

Ideally we would demonstrate a colouring of $\mathbb{R}^{2}$ using less than 7 colours or show that no such colouring is possible.

This article outlines some progress in this direction, namely that if convex polygons are used as a colouring base at least 6 colours are necessary for an excluded distance.

\section{The colouring scheme}

Let us consider a colouring of Euclidean 2-space using polygonal tiles that are convex, (vertices having interior angles strictly less than $\pi$ ), locally finite and all with area greater than some lower bound $l$. Two tiles meet along an entire common edge and three or more tiles meet at vertices. The interior of a single tile is uniformly coloured (we call this the colour of the tile) and points on the boundary of tiles are coloured arbitrarily with the colours of any incident tile. This colouring scheme is exactly the same as with previous lattice-sublattice schemes except that convex tiles are used as a base rather than Voronoi regions.

\section{A lower bound on the chromatic number of the tile based colouring scheme}

LEMMA 3.1. If a colouring is distance excluding then there is a vertex where 3 differently coloured tiles meet.

ProOF. For brevity's sake let us call a vertex where 3 differently coloured tiles meet a tri-coloured vertex.

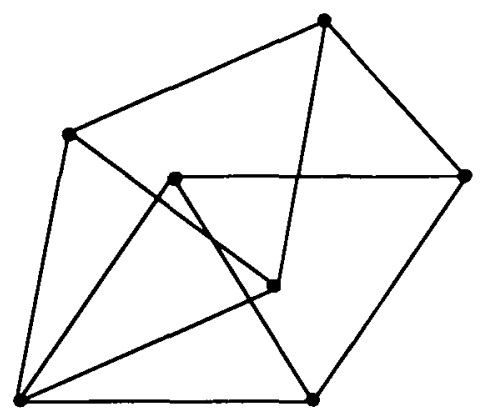

FIGURE I. The Moser spindle 


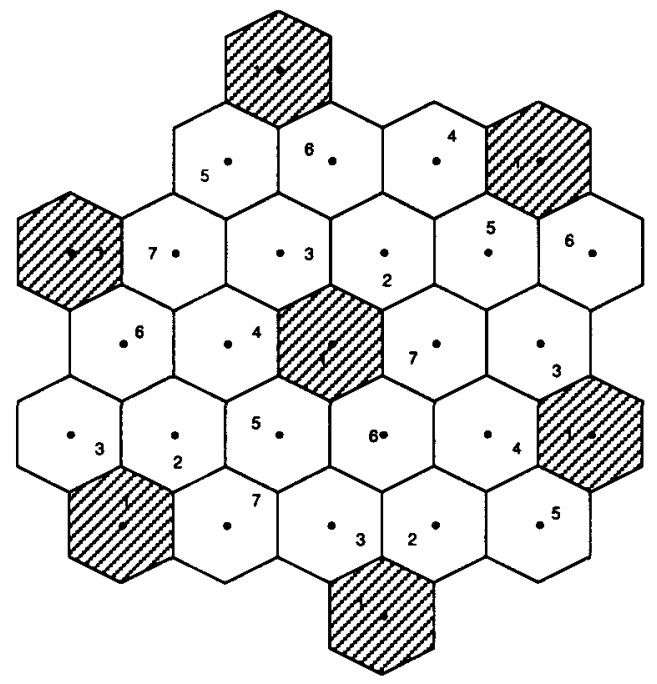

FIGURE 2. The hexagonal lattice 7 colouring

Suppose to the contrary we have an excluded distance $D$ and there is no tri-coloured vertex.

Choose a tile and group together the maximal connected group of like coloured tiles (that includes the one chosen) to form $G$.

If two interior points in this group are distance $D$ apart we have a contradiction, else we continue as follows.

Choose a tile not in $G$ incident with the group $G$ (so necessarily of a different colour) and then expand this to a maximally connected like coloured group to form $M$. This new group $M$ contacts the group $G$ along an entire connected component of the boundary of $G$ (else we would have a tri-coloured vertex). This boundary component is either

- Unbounded, as shown in Figure 3 (a), (which would contradict the existence of the excluded distance $D$ ) and so is not possible,

- Interior to the group, as shown in Figure 3 (b), (there are only finitely many of these groups possible) or

- Exterior to the group, as shown in Figure 3 (c).

- Both interior and exterior to the group $G$.

Now let $G$ become $G \cup M$ and iterate this process (of forming $M$ and then combining to get a new $G$ ).

By finiteness considerations one of the boundary groups $M$ must eventually be exterior (and so be a loop) and have two points distance $D$ apart in which case $D$ cannot be an excluded distance, contradiction. 
Thus an excluded distance $D$ and the lack of a tri-coloured vertex are not possible and the lemma follows.

LEMMA 3.2. A tile based colouring needs at least 5 colours to be distance excluding.

ProOF. We show that if a colouring is distance $D$ excluding then almost all points distance $D$ away from a tri-coloured vertex $T$ (coloured with colours $a, b, c$ ) are coloured with one of at least two different colours ( $d$ and $e$ say).

Consider the points on the circumference $C$ of a circle of radius $D$ with centre $T$ (see Figure 4 (a)). By the finite properties of the tiles all but finitely many of the points on $C$ will be points interior to tiles and by taking a (uniformly coloured) neighbourhood $U$ about each of these points (which will be coloured the same as the circumference point) we see that $U$ cannot be coloured $a, b$ or $c$ if $D$ is to be an excluded distance. Hence almost all points on $C$ need to be coloured a different colour from $a, b$ or $c$.

As almost all points of $C$ are interior it is possible to find two interior points of $C$ that are distance $D$ apart and these must necessarily be coloured differently $d$ and $e$ say, if we are to have $D$ as an excluded distance.

LEMMA 3.3. If a 5 coloured tile based colouring is distance $D$ excluding then all non interior points of $C$ (a circle of radius $D$ with cenre $T$ a tri-coloured vertex) separating interior points of $C$ of different colours are not distance $D$ from an interior point of $C$.

PROOF. Suppose to the contrary that only 5 colours have been employed and we have a non-interior point of $C$ separating two regions of $C$ of different colours that is distance $D$ away from an interior point. The situation depicted in Figure 4 (b). A small perturbation of the line segment of length $D$ (if the interior point is coloured $d$ or $e$ ) gives a contradiction (to the existence of an excluded distance $D$ ).

COROLLARY 3.4. If a 5 coloured tile based colouring is distance $D$ excluding then there must be 6 non interior points of $C$ separating interior points of $C$ of different colours arranged as depicted in Figure 4 (c).

PROof. The arrangement of 6 non interior points as shown above is forced by Lemma 3.3. (Note that there may be other non interior points separating these 6 non interior points.) The existence of one of these non interior points separating an arc coloured $d$ from an arc coloured $e$ is given by Lemmas 3.2 and 3.3. Now the fact that $D$ is an excluded distance forces this to be repeated (in reverse order as we alternate around) for the other 5 points. 


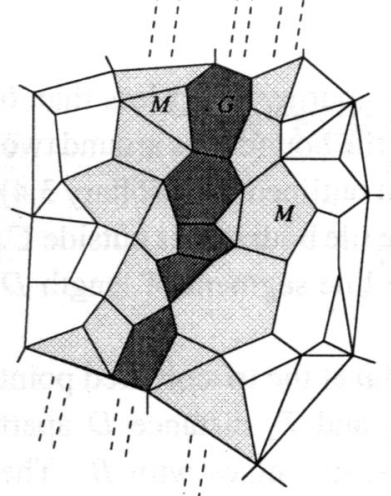

(a)

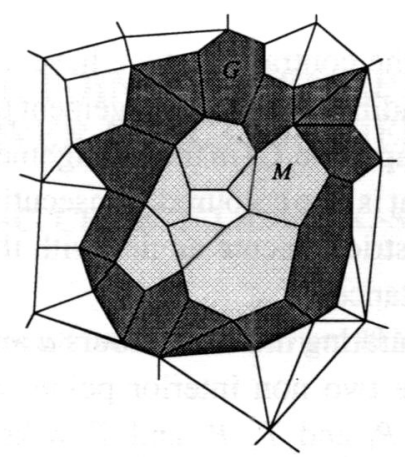

(b)

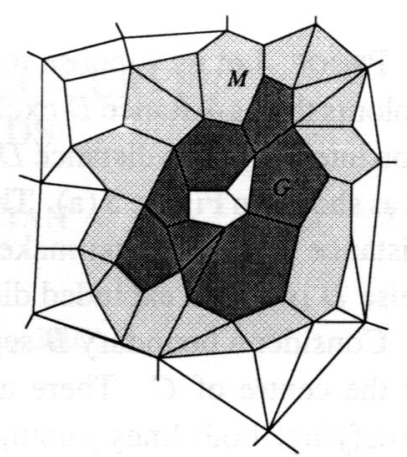

(c)

FIGURE 3.

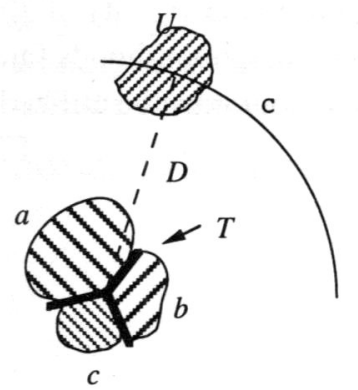

(a)

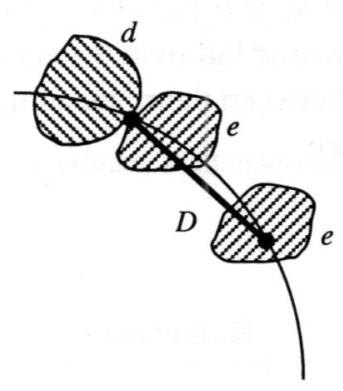

(b)

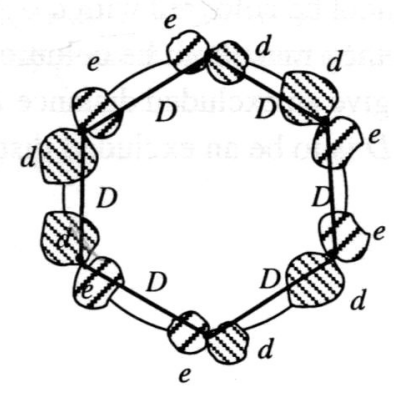

(c)

FIGURE 4.

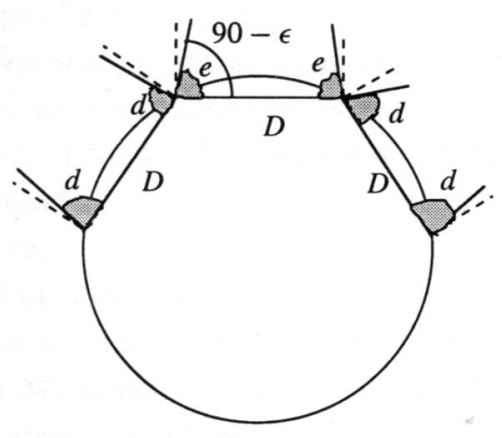

(a)

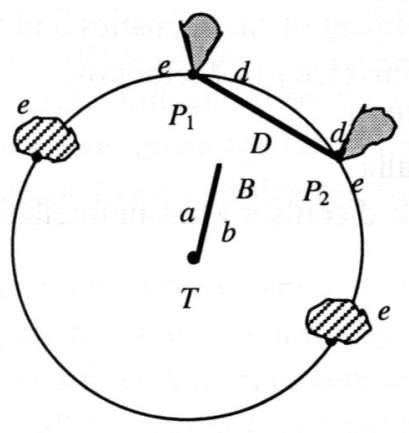

(b)

FIGURE 5. 
THEOREM 3.5. A tile based colouring with excluded distance D must employ at least 6 colours.

PROOF. Let us suppose to the contrary that we have a colouring using less than 6 colours that is distance $D$ excluding then the arrangement of tile boundaries around two non interior points distance $D$ apart on $C$ (in the arrangement outlined in Corollary 3.4) is as shown in Figure 5 (a). That is like coloured, consecutive tile boundaries outside $C$, distance $D$ apart, must make strictly acute angles with the line segment of length $D$ (else $D$ is not an excluded distance).

Consider a boundary $B$ separating the two colours $a$ and $b$ at the tri-coloured point at the centre of $C$. There are two non interior points $P_{1}$ and $P_{2}$ distance $D$ apart satisfying; both lines joining $P_{1}$ and $T, P_{2}$ and $T$ make acute angles with $B$. The situation is depicted in Figure 5 (b).

Now consider the ray like neighbourhoods outside $C$ at $P_{1}$ and $P_{2}$ these cannot be coloured with $d$ or $e$ (else $D$ is not an excluded distance in the vicinity of $C$ ) and they cannot be coloured with $a$ or $b$ (else $D$ is not an excluded distance in the vicinity of $T$ ) so they must both be coloured with $c$ but then these two ray like neighbourhoods fail to give an excluded distance $D$ between them, meaning a sixth colour must be utilized if $D$ is to be an excluded distance.

\section{References}

[1] D. Coulson, 'A 15-colouring of 3-space omitting distance one', Discrete Math. 256 (2002), 83-90.

[2] D. E. Raiskii, 'The realisation of all distances in a decomposition of the space $\mathbb{R}^{n}$ into $n+1$ parts', Math. Notes 7 (1970), 194-196.

Department of Mathematics and Statistics

The University of Melbourne

VIC 3010

Australia

e-mail: d.coulson@ms.unimelb.edu.au 